\title{
An Inertial Sensor for Parts Inspection
}

\author{
S. M. Tam and K. C. Cheung \\ Department of Mechanical Engineering \\ The University of Hong Kong \\ Pokfulam Road, Hong Kong
}

\begin{abstract}
This paper describes the development of a sensor which performs parts inspection by measuring the inertial properties of the part. The sensor comprises a platform supported on a column which is fixed to a rigid base. The part under inspection is attached to the platform to form a single body. By measuring the static deflections of the column and the natural frequency of vibration of the system, it is possible to determine the spatial location of the centre of mass of the part.
\end{abstract}

\section{Introduction}

Inspection is usually required to ensure that a part is machined according to its specification. It often involves measuring various attributes of the part under inspection and comparing the measured values against those specified. The attributes being measured may include the size, shape, mass, locations and sizes of drilled holes and locations and sizes of milled slots of the part.

Human inspection, which measures those attributes manually using vernier callipers, micrometers, balances and gauges, is ineffective and expensive. In order to increase the efficiency of quality inspection, automated inspection systems were introduced. Vision system is commonly used for the automatic inspection of parts [1] .

In a vision system, video cameras are used to capture, from one or more angles, the images of the part under inspection and a computer used for signal analysis. The analysis was done by comparing the images of the part with those of a good part. Therefore, a camera with higher resolution will give a more accurate result. Although high resolution makes the system accurate, a large volume of image data needs to be handled, resulting in long cycle times. Moreover, the quality of the captured images are seriously affected by working conditions such as poor lighting and smoky environment.
Also, internal defects such as presence of internal void cannot be detected by vision system. To overcome the deficiencies of vision-based inspection system, it is desirable to have a sensor which inspects parts by measuring properties of the part other than its appearance.

The fundamental difference between a good part and a defective one lies with their masses and locations of centres of mass. Generally, a defective part will have a different mass as well as a different location of centre of mass compared with a good part, irrespective of the defect. However, depending on the nature of the defect, a defective part may still have the same mass as a good part. An example of such defect is a hole drilled at a wrong location with the same amount of material removed as in a properly machined part. The only difference between a part containing such defects and a good part is in the locations of their centres of mass.

This paper reports on the development of a sensor which measures the location of the centre of mass of a given part. The sensor described here can be easily incorporated with a robotic material handling system so that inspection can be performed during parts handling.

The sensor body consists of three sections : a platform supported on a column and a rigid base to which the column is attached. The part under inspection is placed on the platform. The static deflections of the column are then measured by strain gauges mounted on the column and recorded by a microcomputer. The system, comprising the part plus platform and the column, is then set into free vibration by an impact from a solenoid actuated bolt. The vibration of the column is picked up by the strain gauges and the microcomputer then determines the frequency of vibration which is the natural frequency of the system. The spatial location of the centre of mass can then be calculated from the static deflections and natural frequency of vibration.

The method is described in the section 2. Figure 1 shows a schematic view of the proposed sensor with the 
associated corrdinate system.

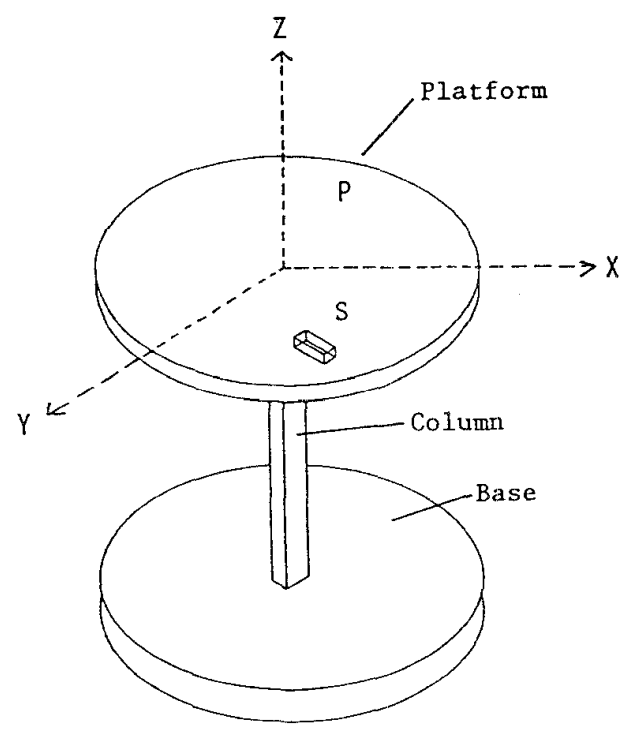

Figure 1. Schematic view of sensor

\section{Nomenclature}

\section{L}

$\mathrm{Xg}, \mathrm{Yg}, \mathrm{Zg}$ Ixx, lyy

$E$ $\theta x s, \theta y s$

$I_{M X}, I_{M Y}$

$\mathrm{m}$

$\operatorname{mp}$

mc

fy

$\mathrm{Kx}, \mathrm{Ky}$

$\mathrm{Kxx}$

Icx length of column

coordinates of the centre of mass second moment of area of the crosssection of the column

Young's modulus of the column static deflections of column in the $x$ and $y$ axes respectively moments of inertia of the test piece about the $\mathrm{x}$ and $\mathrm{y}$ axes respectively mass of test piece mass of platform mass of column natural frequency of the system when vibrating within the $x-z$ plane bending stiffiness of column at $x$ and $y$ axes respectively

linear stiffness of column at the $x$ axis moment of inertia of sensor about the $x$ axis

\section{Theory}

This section outlines the methods for obtaining the coordinates of the centre of mass ( $\mathrm{Xg}, \mathrm{Yg}, \mathrm{Zg}$ ) of an object placed on the platform of the sensor. The microcomputer then uses this data to distinguish defective parts from good ones.

\subsection{Determination of $\mathrm{Xg}$ and $\mathrm{Yg}$}

When an object is placed on the platform of the sensor, the static deflections of the column, $\theta_{X S}$ and $\theta_{Y S}$, are given by the static equilibrium equation below.

$$
\left(\begin{array}{l}
K x \\
K y
\end{array}\right) \cdot\left(\begin{array}{l}
\theta_{X S} \\
\theta_{Y S}
\end{array}\right)=\left(\begin{array}{l}
-m g X g \\
-m g Y g
\end{array}\right)
$$

where $\mathrm{Kx}$ and $\mathrm{Ky}$ are the bending stiffness of the column in the $\mathrm{x}$ and $\mathrm{y}$ axes respectively.

$$
K x=\frac{3 E I_{X X}}{\left(L+Z_{g}\right)^{2}} \quad K_{Y}=\frac{3 E I_{Y Y}}{\left(L+Z_{g}\right)^{2}}
$$

$\mathrm{Xg}$ and $\mathrm{Yg}$ can then be calculated by rearranging equation (1) into:

$\left(\begin{array}{l}X g \\ Y g\end{array}\right)=-\frac{1}{m g} \cdot\left(\begin{array}{l}K x \\ K y\end{array}\right) \cdot\left(\begin{array}{l}\theta x s \\ \theta y s\end{array}\right)$

The static deflections, $\theta_{X S}$ and $\theta_{Y S}$, are measured by strain gauges mounted on the column as show in Figure 2.

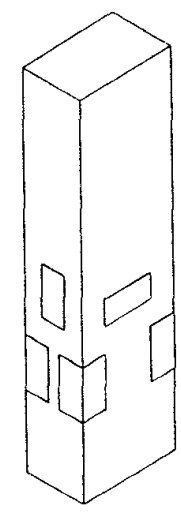

Figure 2. Strain gauges mounted on the column 


\subsection{Determination of $\mathrm{Zg}$}

$\mathrm{Zg}$ can be obtained from the characteristic equation of an elastic pendulum.

The characteristic equation of the sensor is derived using the Energy Method which is suitable for analyzing systems under free-vibration.

The Kinetic Energy ( Tx ) of the column when vibrating within the $x-z$ plane which is given by [ 2 ].

$T_{X}=\frac{1}{2} I_{C X} \dot{\theta}_{X S}^{2}+\frac{1}{2}\left(I_{M X}+m X_{g}^{2}\right) \dot{\theta}_{X S}^{2}+\frac{1}{2} m\left(L+Z_{g}\right)^{2} \dot{\theta}_{X S}^{2}$

The Potential Energy ( $\mathrm{Vx}$ ) stored in the column is given by $[2]$ as

$V_{X}=\frac{1}{2} K_{X X}\left[\left(L+Z_{g}\right) \theta_{X S}\right]^{2}$

where

$$
K_{X X}=\frac{3 E I_{X X}}{\left(L+Z_{g}\right)^{3}}
$$

is the linear stiffness

of the column.

Therefore,

$Y_{X}=\frac{1}{2}\left[\frac{3 E I_{X X}}{\left(L+Z_{z}\right)}\right] \cdot \theta_{X S}^{2}$

Free vibration involves the cyclic interchange of kinetic energy and potential energy. For free vibration with negligible damping, it can be assumed that no energy is dissipated. Therefore, the sum of kinetic energy and potential energy remains constant.

$$
T_{X}+V_{X}=\text { constant }
$$

Therefore,

$$
\frac{d}{d t}\left(T_{X}+V_{X}\right)=0
$$

Substituting yields

$\left[I_{c X}+I_{M X}+m X_{g}^{z}+m\left(L+Z_{g}\right)^{2}\right] \ddot{\theta}_{X S} \dot{\theta}_{X s}+\frac{3 E I_{X X}}{\left(L+Z_{g}\right)} \dot{\theta}_{X S} \theta_{X s}=0$

The above equation can be expressed as :

$I \cdot \ddot{\theta}_{x s}+K \cdot \theta_{x s}=0$ where

$$
I=\left[I_{C X}+I_{M X}+m X_{g}^{2}+m\left(L+Z_{g}\right)^{2}\right]
$$

and

$$
K=\frac{3 E I_{X X}}{\left(L+Z_{g}\right)}
$$

Equation ( 3 ) is a typical undamped free-vibration equation with natural frequency.

$$
f_{y}=\frac{1}{2 \pi} \sqrt{\frac{K}{I}}
$$

Substituting for $\mathrm{K}$ and I gives

$f_{y}=\frac{1}{2 \pi} \sqrt{\frac{3 E I_{X X}}{m\left(L+Z_{g}\right)^{3}+\left(I_{C X}+I_{M X}+m X_{g}^{2}\right)\left(L+Z_{g}\right)}}$

where $I_{C X} \cong\left(\frac{33}{140} \cdot m_{C}+m_{P}\right) \cdot L^{2}$

Normally, $\ell_{M X}$ is small compared with other terms. Therefore, it can be ignored in the calculation. Thus, the equation can be revised as follows :

$$
f_{y}=\frac{1}{2 \pi} \sqrt{\frac{3 E I_{x x}}{m\left(L+Z_{g}\right)^{3}+\left[\left(\frac{33}{140} m_{C}+m_{p}\right) L^{2}+m X_{g}^{2}\right]\left(L+Z_{g}\right)}}
$$

fy is the measured natural frequency of vibration of the system. Being the only unknown in the above equation, $\mathrm{Zg}$ can then be calculated using a numerical root-finding routine such as the Van Wijngaarden-Dekker-Brent method [3]

\section{Mechanical hardware}

The prototype sensor was made of Dural $79-\mathrm{TF}$ aluminum alloy. The material has high ultimate tensile strength and low damping. The sensor was machined out of a single block of metal in order to avoid backlash and multi-degree-of-freedom vibration. The platform diameter was chosen as $100 \mathrm{~mm}$ with a thickness of $3 \mathrm{~mm}$. This platform design provides sufficient strength to support the largest test piece without deformation and enough rigidity to enable the column to be considered as the only elastic element. The setup is considered as a two degree-offreedom vibratory system. 
The base of the prototype sensor was firmly bolted onto heavy steel plates so that movement of the end of the column is negligible.

\section{Electronic hardware}

Being accurate, inexpensive, and easily installed, strain gauges were selected as the primary sensing elements. They were mounted on the column and connected into a Wheatstone bridge for signal processing. The output signals from the Wheatstone bridge were amplified by instrumentation amplifiers.

When measuring the natural frequency of vibration of the system, Phase-Locked-Loop (PLL) circuits were used to convert the amplified signals to square waves so that calculation of frequency is simpler and more accurate. A digital counter is used to measure the natural frequency of vibration of the system.

The signals are fed to a microcomputer via a Data Acquisition Card consisting of analog-to-digital converters and digital-to-analog converters as well as a digital counter for frequency measurement.

Figure 3 shows the arrangement of the signal processing hardware.

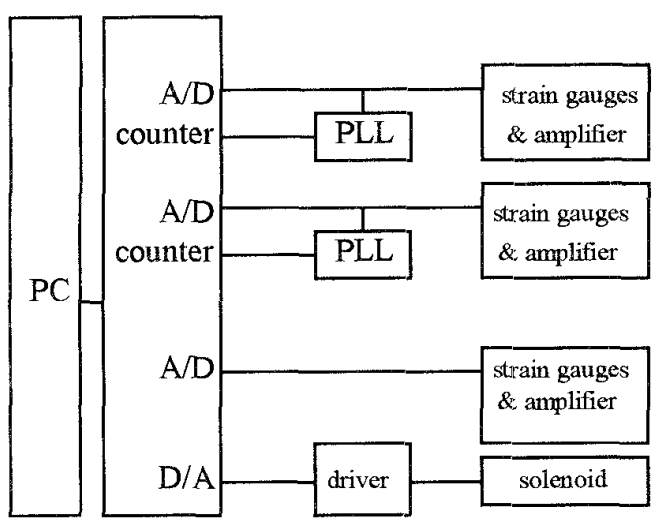

Figure 3. Block diagram of the signal processing hardware

\section{Software}

The sensor is controlled by a program written in $\mathrm{C}++$ which consists of four parts. Part 1 performs static measurement and computation to obtain the coordinates $\mathrm{Xg}$ and $\mathrm{Yg}$ of a part. Part 2 implements the control function which sends a signal to switch on the solenoid actuated bolt to produce an impact to the platform and sets the system into free vibration. Parts 3 and 4 carry out the frequency counting and calculation of $\mathrm{Zg}$.

\section{Operation}

The part $S$ is placed onto platform $P$ and held firmly by a magnetic sheet. The purpose is to ensure that $S$ and $\mathrm{P}$ act like a single rigid body. This does not limit the use of the sensor to ferrous objects because non-ferrous objects can be fixed onto the platform by means of jig and jaws. The platform is rigid while the column is elastic. Three sets of strain gauges mounted on the column as shown in Figure 2 measure the static deflections of the column. The system is then set into free vibration by an impact from the solenoid actuated bolt. The free vibration of the sensor body with the object is measured by the strain gauges, then amplified and converted to square waves. The I/O interface circuit determines the frequency of vibration. The microcomputer then uses the static deflections and the natural frequency of vibration to calculate the spatial location of the centre of mass of the object.

\section{Experimental results}

In order to evaluate the accuracy of measurement of the sensor, three test pieces of different dimensions were manufactured. The location of the centre of mass of each of the test piece was determined using the sensor.

The calculated coordinates of the centres of mass were compared with the actual values as shown in Table 1 below.

\begin{tabular}{|c|c|c|c|}
\hline Specimen no. & 1 & 2 & 3 \\
\hline Mass $(\mathrm{g})$ & 463 & 454 & 501 \\
\hline Dimension $(\mathbf{m m})$ & $\varnothing 60 \times 40$ & $\varnothing 43 \times 40$ & $40 \times 40 \times 40$ \\
\hline Material & $\begin{array}{l}\text { Aluminum } \\
\text { alloy }\end{array}$ & Mild steel & Mild steel \\
\hline $\begin{array}{l}\text { Actual value of } \\
\mathrm{Zg}(\mathrm{mm})\end{array}$ & 20 & 20 & 20 \\
\hline $\begin{array}{l}\text { Experimental } \\
\text { value of } \mathrm{Zg} \\
\text { (mm) }\end{array}$ & 20.39 & 19.92 & 19.46 \\
\hline Error in $\mathrm{mm}$ & +0.39 & -0.08 & -0.34 \\
\hline Error in \% & 1.987 & 0.395 & 2.681 \\
\hline $\begin{array}{l}\text { Actual value of } \\
\mathrm{Xg}(\mathrm{mm})\end{array}$ & 30 & 21.5 & 20 \\
\hline $\begin{array}{l}\text { Experimental } \\
\text { value of } \mathrm{Xg} \\
(\mathrm{mm})\end{array}$ & 29.75 & 21.7 & 20.27 \\
\hline Error in $\mathrm{mm}$ & -0.25 & +0.2 & +0.27 \\
\hline Error in \% & 0.833 & 0.93 & 1.35 \\
\hline $\begin{array}{l}\text { Actual value of } \\
\mathrm{Yg}(\mathrm{mm})\end{array}$ & 30 & 21.5 & 20 \\
\hline $\begin{array}{l}\text { Experimental } \\
\text { value of } \mathrm{Yg} \\
(\mathrm{mm})\end{array}$ & 30.28 & 21.2 & 20.28 \\
\hline Error in mm & +0.28 & -0.3 & +0.28 \\
\hline Error in $\%$ & 0.933 & 1.395 & 1.38 \\
\hline
\end{tabular}

locations of centres of mass. 
As can be seen from Table 1, the calculated values of $\mathrm{Xg}, \mathrm{Yg}$ and $\mathrm{Zg}$ were all within $\pm 0.4 \mathrm{~mm}$ of the actual values. This shows that the proposed sensor is capable of measuring, to an accuracy of less than $\pm 0.4 \mathrm{~mm}$, the spatial location of the centre of mass of an object placed on the platform.

\section{Conclusion}

A prototype sensor for detecting the location of the centre of mass of a part has been developed. By comparing the measured location with that of a known good part, the system can discriminate between good and defective parts. The sensor has three features. First, it operates by measuring parameters related to the inertia of the part. Second, it incorporates an inexpensive personal computer. Third, the calculation of $\mathrm{Zg}$ ( centre of mass of a part in the Z-direction) is based on a new equation. The equation is useful in calculating the natural frequency of complex pendulum system.

Three advantages of the sensor are that, unlike vision systems, it is not affected by poor lighting, dust and fumes, it can detect defects such as internal holes which would escape detection by vision-based inspection system, it is also inexpensive compared with vision system.

The accuracy of measurement is affected by the design of the sensor such as the length and cross-sectional dimension of the column as well as the position of the object on the platform relative to the neutral axes of the column. Further investigation is therefore needed to establish the relationship between the accuracy of measurement and the above factors. It is then possible to achieve an optimal design for the sensor.

Also, the operation of the sensor requires the knowledge of the mass of the part. Certain defects, such as presence of internal void, causes a change in the mass of the object as well as a change in the location of its centre of mass. Therefore, a sensor capable of measuring both the mass and the spatial location of the centre of mass of an object placed on the platform of the sensor will be developed.

\section{Acknowledgements}

One of authors, S.M Tam, wishes to thank his mother, Mrs Ying Lin Chan, for her unstinting faith and patience. The research began in November 1993 in the Department of Mechanical Engineering, University of Hong Kong. The project is financially supported by the Committee on Research and Conference Grants of the University of Hong Kong.

\section{References}

[1] Pau L.F., Computer Vision for Electronics Manufacturing, Plenum Press, 1989, Page 40 and 148.

[2] Beards C.F., Structural Vibration Analysis, Halsted Press, 1980, John Wiley \& Sons.

[3] Brent, D. Algorithms for minimization without derivatives, Chapter 3 and 4, Englewood Cliffs, New Jersey, Prentice Hall 1973. 